\section{CARDIOVASCULAR MAGNETIC RESONANCE LEFT VENTRICULAR STROKE VOLUME AND OUTCOME PREDICTION IN PATIENTS WITH AORTIC STENOSIS FOLLOWING INTERVENTION}

'Subothini Selvendran*, ${ }^{1}$ Nikhil Aggarwal, ${ }^{2}$ Dominique Auger, ${ }^{2}$ Eva Nyktari, ${ }^{2}$ Claire Raphael, ${ }^{3}$ Sanjay Prasad, ${ }^{3}$ Vass Vassiliou. ${ }^{1}$ Imperial College London; ${ }^{2}$ RBH; ${ }^{3}$ Royal Brompton Hospital; " Presenting Author

\subsection{6/heartjnl-2016-309890.41}

Introduction Percutaneous transcatheter aortic valve implantation (TAVI) is gaining significant popularity as an alternative to conventional aortic valve replacement surgery for patients with aortic stenosis (AS), especially in high operative risk patients. We investigated outcome prediction using a cohort of patients undergoing TAVI and utilising patient demographic and cardiovascular magnetic resonance (CMR) parameters.

Methods Between 2010-2014, 100 consecutive patients with severe AS scheduled for TAVI underwent CMR. We retrospectively analysed data on a variety of patient demographics to identify any associations with overall mortality. Follow-up was completed using electronic hospital records and data obtained from the National Strategic Office. Kaplan-Meier curves were obtained and compared using the log-rank test. Univariate analysis was used on 20 variables and those significant included in a multivariate Cox regression model.

Results 100 patients (median age 81 years; 55 male) were followed for a median 1349 days. During this period 20 patients died. 12 patients were lost to follow up and were censored for statistical purposes at their last known time being alive. On univariate analysis left ventricular stroke volume (LVSV) and known history of hypertension were associated with survival (Table 1 and Figure 1). On multivariable analysis both remained significantly associated with survival $[\mathrm{HR}=2.997$ (95\% CI:1.393-6.448, p $=0.005$ ) for history of hypertension and $\mathrm{HR}=0.983$ (95\% CI:0.968-0.998, $\mathrm{p}=0.031)$ for $1 \mathrm{~mL}$ LVSV increase].

Conclusions In this cohort of elderly AS patients who underwent CMR before TAVI, a lower LV Stroke volume and the presence of hypertension were associated with worse prognosis. LV Stroke Volume appears to be more useful than Left Ventricular Ejection Fraction and Right Ventricular Ejection Fraction. We recommend that it could be routinely assessed in addition to the other parameters already employed in clinical practice.

\section{GENDER DIFFERENCES IN RESPONSE TO TRANSCATHETER AORTIC VALVE IMPLANTATION IN PATIENTS WITH SEVERE AORTIC STENOSIS ASSESSED BY FEATURE TRACKING}

${ }^{1}$ James RJ Foley*, ${ }^{2}$ Laura E Dobson, ${ }^{2}$ Tarique A Musa, ${ }^{2}$ Timothy A Fairbairn, ${ }^{2}$ Akhlaque Uddin, ${ }^{2}$ Peter P Swoboda, ${ }^{2}$ Pankaj Garg, ${ }^{2}$ Graham J Fent, ${ }^{2}$ Philip Haaf, ${ }^{3}$ Christopher J Malkin, ${ }^{3}$ Daniel J Blackman, ${ }^{3}$ Sven Plein, ${ }^{3}$ John P Greenwood. ' University of Leeds; ${ }^{2}$ LICAMM \& MCRC, University of Leeds; ${ }^{3}$ Leeds Teaching Hospitals Trust; *Presenting Author

\subsection{6/heartjnl-2016-309890.42}

Introduction Aortic stenosis (AS) results in left ventricular hypertrophy, myocyte dysfunction and fibrosis as a physiological response to excessive afterload. Transcatheter Aortic Valve implantation (TAVI) is an effective treatment for severe AS, however controversy exists whether gender predisposes to

Abstract 41 Table 1 Demonstrating the variables analysed and their results in univariate and bivariate models

\begin{tabular}{|c|c|c|c|c|c|c|}
\hline \multirow[t]{2}{*}{ Parninteter. } & Pvalue & Harard rotlo & $95 \times \mathrm{C}$ & Pvalue & Harard ratlo & $95 \% \mathrm{C}$ \\
\hline & \multicolumn{3}{|c|}{ Unwarlate } & \multicolumn{3}{|c|}{ Bivarlable } \\
\hline Age & 0.231 & & & & & \\
\hline Gender & 0.219 & & & & & \\
\hline Weight & 0.337 & & & & & \\
\hline Atrial Fibrillation & 0.333 & & & & & \\
\hline Diabetes Mellitus & 0.127 & & & & & \\
\hline History of Hypertension & 0.019 & 2.415 & $1.155-5.050$ & 0.005 & 2.997 & $1.393-6.448$ \\
\hline $\begin{array}{l}\text { Previous Myocardial } \\
\text { Infarction }\end{array}$ & 0.305 & & & & & \\
\hline Systolic Blood Pressure & 0.560 & & & & & \\
\hline Diastolic Blood Pressure & 0.330 & & & & & \\
\hline NYHA Class & 0.439 & & & & & \\
\hline Creatinine & 0.310 & & & & & \\
\hline Estimated PAP & 0.971 & & & & & \\
\hline Peak AV gradient & 0.590 & & & & & \\
\hline เV Mass & 0.995 & & & & & \\
\hline LV EOV & 0.208 & & & & & \\
\hline IVEF & 0.77 & & & & & \\
\hline เV SV & 0.010 & 0.978 & $1.393-6.448$ & 0.031 & 0.983 & $0.968-0.998$ \\
\hline RVEDV & 0.128 & & & & & \\
\hline RVEF & 0.250 & & & & & \\
\hline RVSV & 0.182 & & & & & \\
\hline
\end{tabular}




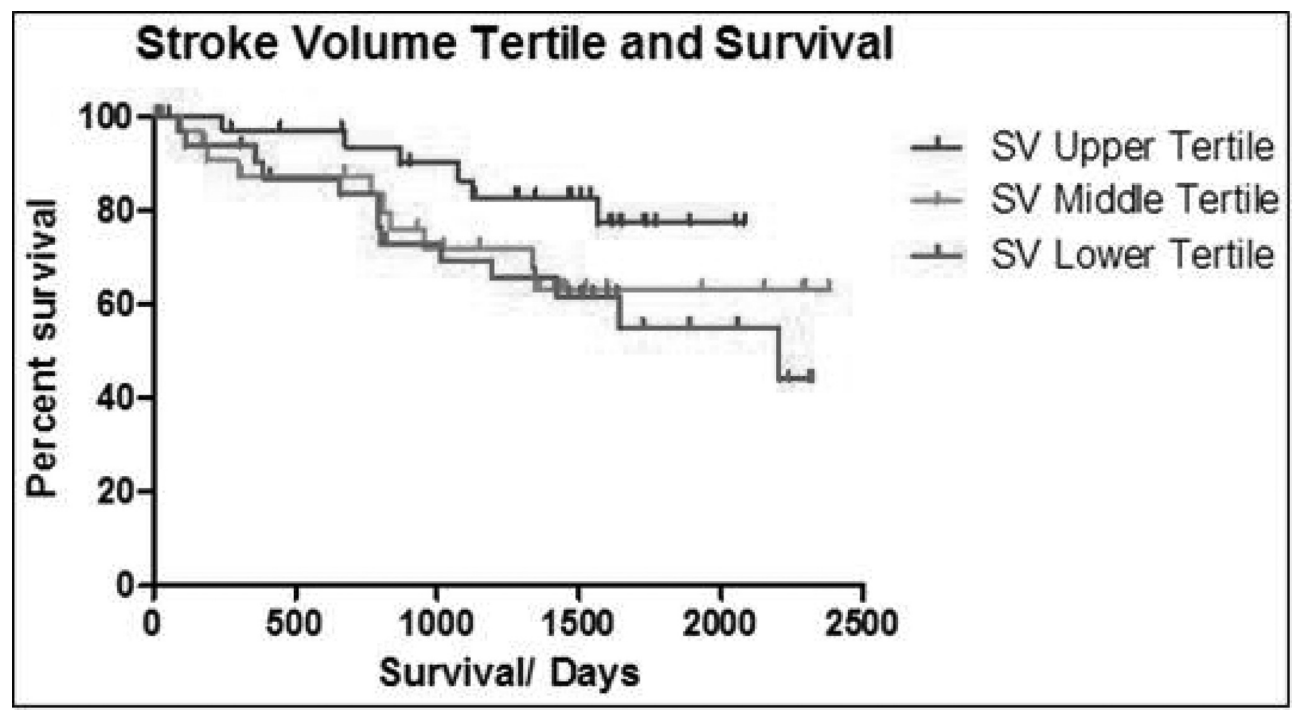

Abstract 41 Figure 1 A Kaplan-Meir Estimator Plot illustrating survival according to the LV Stroke Volume Tertiles. Patients in the Upper Tertile had significantly better outcome compared to patients in the Lower Tertile $[\mathrm{HR}=0.37,95 \% \mathrm{Cl}=0.1431-0.9652, \mathrm{p}=0.03$ ]

improved myocardial function post procedure. Feature-tracking cardiovascular magnetic resonance (FT-CMR) is a novel method for quantification of myocardial strain and strain rate that can identify subtle changes in left ventricular (LV) mechanics prior to overt changes in LV ejection fraction (EF). Our aim was to compare the acute impact of TAVI on LV function between males and females defined by strain measurements.

Methods 59 patients, 34 males and 25 females matched for age $(78.4 \pm 7.19$ years vs $78.8 \pm 9.7$ years $\mathrm{p}=0.876)$ EuroSCORE II $(5.19 \pm 4.33 \%$ vs $4.26 \pm 2.99 \% \mathrm{p}=0.788)$ and presence of baseline myocardial fibrosis $(p=0.829)$ with severe, symptomatic AS were prospectively enrolled prior to TAVI. All patients underwent an identical 1.5T CMR protocol (Intera or Ingenia, Phillips Healthcare, Best, The Netherlands) at baseline and at a median of 4 days following TAVI. Endocardial and epicardial borders were traced manually on the end-diastolic slice, then strain and strain rate measurements were obtained using commercially available post-processing software (CVI42, Circle Cardiovascular Imaging, Calgary, Alberta, Canada).

Results TAVI was associated with a significant and comparable reduction in peak aortic pressure gradient and LV mass index in both men and women (Table 1). There was no significant difference in extent of pressure drop $(\mathrm{p}=0.374)$ or extent of regression of LV mass index $(p=0.095)$ between men and women. There was no significant change in left ventricular end diastolic volume indexed (LVEDVI) or LVEF observed acutely following TAVI, in men or women (Table 1). Longitudinal strain $\left(\mathrm{E}_{\mathrm{ll}}\right)$, circumferential strain $\left(\mathrm{E}_{\mathrm{cc}}\right)$ and circumferential strain rate $\left(\mathrm{SR}_{\mathrm{cc}}\right)$ did not change significantly in men or women (Table 2). However, TAVI was associated with a significant increase in longitudinal strain rate $\left(\mathrm{SR}_{\mathrm{ll}}\right)$ in males $(\mathrm{p}=$ 0.012 ) that was not observed in females $(p=0.184)$ (Table 2).

Conclusion TAVI was associated with structural reverse remodelling that was comparable between males and females. Functional recovery in LV mechanics following TAVI may occur earlier in males than in females, although this may reflect the higher LVMI that men have at baseline prior to TAVI.

\section{Interventional Cardiology}

\section{EUROSCORE II AND STS RISK MODEL SCORES IN AORTIC STENOSIS: CAN WE RELY ON THEM?}

${ }^{1}$ Nikhil Aggarwal ${ }^{*},{ }^{1}$ Subothini Selvendran, ${ }^{2}$ Simon Newsome, ${ }^{3}$ Tamir Malley, ${ }^{3}$ Dominique Auger, ${ }^{3}$ Sanjay Prasad, ${ }^{3}$ Vass Vassiliou. ${ }^{1} /$ mperial College London; ${ }^{2}$ London School of Hygiene and Tropical Medicine; ${ }^{3} \mathrm{RBH}$; *Presenting Author

\subsection{6/heartjnl-2016-309890.43}

Introduction The European System for Cardiac Operative Risk Evaluation II (EuroSCORE) and Society of Thoracic Surgeons (STS) risk models provide a method of predicating mortality of patients undergoing cardiac surgery. However, their validity in transcatheter aortic valve implantation (TAVI) remains controversial with some studies supporting its use as a good predictor of mortality whilst others find no association. We sought to investigate the validity of both EuroSCORE II and STS score as predictors of mortality in a real-life cohort of patients undergoing a TAVI.

Methods Between 2010-2014, 115 (79 \pm 8 years old; 56 male) consecutive patients with severe AS scheduled for TAVI had EuroSCORE and STS score calculated prior to

\begin{tabular}{llll} 
Abstract 42 Table 1 & Patient characteristics & \\
\hline & Pre-TAVI & Post-TAVI & p-value \\
\hline PPG male $(\mathrm{mmHg})$ & $89.5 \pm 22.6$ & $17.3 \pm 8.6$ & $<0.001$ \\
PPG female $(\mathrm{mmHg})$ & $88.1 \pm 21.8$ & $15.8 \pm 7.6$ & $<0.001$ \\
LVMI male $\left(\mathrm{g} / \mathrm{m}^{2}\right)$ & $77.2 \pm 16.6$ & $68.8 \pm 15.5$ & $<0.001$ \\
LVMI female $\left(\mathrm{g} / \mathrm{m}^{2}\right)$ & $74.5 \pm 15.6$ & $67.4 \pm 14.3$ & $<0.001$ \\
LVEF male $(\%)$ & $53.2 \pm 13.2$ & $53.6 \pm 12.2$ & 0.611 \\
LVEF female $(\%)$ & $55.0 \pm 13.8$ & $55.9 \pm 12.2$ & 0.528 \\
LVEDVI male (ml $\left./ \mathrm{m}^{2}\right)$ & $101.0 \pm 26.0$ & $99.9 \pm 23.2$ & 0.650 \\
LVEDVI female $\left(\mathrm{ml} / \mathrm{m}^{2}\right.$ ) & $96.1 \pm 24.1$ & $93.3 \pm 20.1$ & 0.204 \\
\hline PPG - peak pressure gradient & & \\
LVMI - left ventricular mas indexed & & \\
LVEDVI - left ventricular end diastolic volume indexed & & \\
LVEF - left ventricular ejection fraction & &
\end{tabular}

